\title{
EP-156
}

\section{Prophylactic octreotide according to individual risks for postoperative pancreatic fistula after pancreatoduodenectomy}

\author{
So Jeong YOON, In Woong HAN*
}

Division of Hepatobiliary-Pancreatic Surgery, Department of Surgery, Samsung Medical Center, Sungkyunkwan University School of Medicine, Seoul, Korea

Introduction: Postoperative pancreatic fistula (POPF) is one of the most critical complications following pancreatic surgery. The aim of this study was to evaluate selective use of prophylactic octreotide for patients stratified by their individual risks of POPF.

Methods: From June 2019 to July 2020, a total of 243 patients underwent pancreatoduodenectomy with pancreatojejunostomy in Samsung Medical Center. Individual POPF risk scores were calculated with previously developed nomogram and clinicopathological data of the patients were retrospectively reviewed.

Results: There were 81 patients in low-risk group and 182 patients in high-risk group. No statistically significant differences were found in clinically relevant POPF (CR-POPF) rates between the control group and the octreotide group in both low- $(3.0 \%$ vs. $7.1 \%, p=$ $0.439)$ and high- $(23.6 \%$ vs. $16.1 \%, p=0.206)$ risk groups. In risk factor analyses, postoperative use of octreotide was not an independent risk factor for CR-POPF in all $(p=0.897)$ patients, low- $(p=0.436)$, and high- $(p=0.614)$ risk group. Drain fluid amylase on the first postoperative day was significantly correlated with CR-POPF, regardless of individual risks.

Conclusions: Selective use of octreotide, even in high risk patients, showed no protective effect against POPF. Routine use of postoperative octreotide would not be recommended. 